\title{
RELAÇÃO ENTRE OCORRÊNCIA DE ENDOMETRIOSE E SOFRIMENTO PSIQUÍCO
}

\section{Relationship between occurrence of endometriosis and psychic suffering Relación entre la endometriosis y el sufrimiento psíquico}

\author{
Luis Adriano Freitas Oliveira \\ Universidade de Fortaleza - UNIFOR - Fortaleza (CE) - Brasil
}

Aline Veras Morais Brilhante

Universidade de Fortaleza - UNIFOR - Fortaleza (CE) - Brasil

Lidia Andrade Lourinho

Centro Universitário Farias Brito - FBUni - Fortaleza (CE) - Brasil

\section{RESUMO}

Objetivo: Compreender a relação existente entre a ocorrência de endometriose e o sofrimento psíquico presente nas mulheres. Métodos: Estudo qualitativo realizado com 11 mulheres com endometriose no seu primeiro atendimento em ambulatório de endometriose de uma maternidade de referência em Fortaleza, Ceará, de setembro de 2016 a janeiro de 2017. A coleta de dados ocorreu pelas entrevistas narrativas. Após leitura e transcrição minuciosa das entrevistas, extraiu-se uma categoria para análise. Resultados: A busca incessante pelos serviços de saúde no tratamento das dores incapacitantes são condições que contribuem para a alteração do humor da mulher. A demora no diagnóstico e intervenção leva à mulher a frustração e, frequentemente, a reação estressante. Mesmo após o diagnóstico e a instituição do tratamento, os fatores psicológicos que fazem parte da síndrome dolorosa, como a mudança no humor e a ansiedade, contribuem diretamente para melhorar ou piorar o estado de saúde da mulher. E ainda necessitam de uma abordagem holística para visibilidade dos sintomas, precisando de um suporte emocional para lidar com a doença. Conclusão: A qualidade de vida, valor atribuído à duração da vida quando esta é transformada pela percepção de limitações físicas, psicológicas, funções sociais e oportunidades motivadas pela doença, tratamento e outros agravos, deve ser um aspecto observado no tratamento de pacientes com endometriose, sobretudo quando se consideram a influência dos aspectos psíquicos no tratamento da doença biológica. Em concordância com este sentido sobre a percepção da saúde, as funções sociais, psicológicas e físicas das mulheres com endometriose necessitam ser repensadas e reconfiguradas.

Descritores: Endometriose; Saúde Pública; Sofrimento Psíquico.

\section{ABSTRACT}

Objective: To understand the relationship between the occurrence of endometriosis and the psychic suffering present in women. Methods: A qualitative study conducted with 11 women with endometriosis during their first appointment at the endometriosis outpatients clinic of a referral maternity hospital in Fortaleza, Ceará, from September 2016 to January 2017. Data collection was carried out through narrative interviews. After reading and transcribing the interviews in detail, a category was extracted for analysis. Results: The tireless search for health care services for treatment of disabling pain is a condition that contributes to women's mood swings. The delay in diagnosis and intervention lead women to frustration and, frequently, to stress. Even after diagnosis and the beginning of treatment, the psychological effects comprised in the painful syndrome, such as the mood swings and anxiety, contribute directly to the recovery or worsening of the woman's health. A holistic approach to these women is needed for visibility of the symptoms, and emotional support is required for them to deal with this disease. Conclusion: The quality of life, a value attributed to the duration of life when this is transformed by the perception of physical and psychological limitations, and impaired social functions and opportunities motivated by the disease, treatment, and other aggravations, is an aspect should be observed in the treatment of patients with endometriosis, particularly when the influence of psychic aspects on the treatment of the biological disease is considered. Consistent with this sense of health perception, the social, psychological and physical functions of women with endometriosis need to be rethought and reconfigured.

Descriptors: Endometriosis; Public Health; Psychological Stress. 


\section{RESUMEN}

Objetivo: Comprender la relación entre la endometriosis y el sufrimiento psíquico de mujeres. Métodos: Estudio cualitativo realizado con 11 mujeres con endometriosis en su primer visita al ambulatorio de endometriosis de una maternidad de referencia de Fortaleza, Ceará entre septiembre de 2016 y enero de 2017. La recogida de datos se dio por entrevistas narrativas. Después de la lectura y la transcripción detallada de las entrevistas, se retiró una categoría para análisis. Resultados: La búsqueda incesante por los servicios de salud para tratamiento de los dolores incapacitantes son condiciones que contribuyen para el cambio de humor de la mujer. El retraso para el diagnóstico y la intervención lleva la frustración de la mujer y, con frecuencia, una reacción estresante. Mismo después del diagnóstico y el inicio del tratamiento, los factores psicológicos del síndrome doloroso como el cambio del humor y la ansiedad contribuyen directamente para la mejoría o el empeoramiento del estado de salud de la mujer. Las mujeres todavía necesitan de un abordaje holístico para la visibilidad de los síntomas requiriendo un soporte emocional para el manejo de la enfermedad. Conclusión: La calidad de vida, valor de la duración de vida cuando esa es transformada por la percepción de las limitaciones físicas y psicológicas, las funciones sociales y las oportunidades motivadas por la enfermedad, el tratamiento y otros agravios, debe ser un aspecto observado en el tratamiento de pacientes con endometriosis, sobre todo cuando se considera la influencia de los aspectos psíquicos en el tratamiento de la enfermedad biológica. De acuerdo a la percepción de la salud se necesita reconsiderar y redistribuir las funciones sociales, psicológicas y físicas de las mujeres con endometriosis.

Descriptores: Endometriosis; Salud Pública; Estrés Psicológico.

\section{INTRODUÇÃO}

A endometriose é uma doença em que há presença de tecido endometrial fora do útero, o que causa uma resposta inflamatória. Pesquisas mostram que as doenças inflamatórias como a endometriose podem estar associadas às doenças psíquicas devido à interação entre sistema imunológico e sistema nervoso central( ${ }^{(1)}$. A endometriose está associada à depressão e à ansiedade, prejudicando, assim, a qualidade de vida das mulheres acometidas.

No então, algumas lacunas estão diretamente relacionadas à endometriose, trazendo um conceito multidimensional que engloba aspectos físicos, psicológicos e sociais ligados a uma doença ou tratamento em particular, o que poderia trazer grandes benefícios aos pacientes e constituir uma excelente intervenção terapêutica ${ }^{(2)}$.

Descobrir a doença é, sem dúvida, um importante evento na vida emocional das mulheres com endometriose, assim como obter o diagnóstico rápido e preciso é fundamental para se intervir, melhorando as estratégias de enfrentamento do sofrimento e da angústia ao aguardar respostas para o tratamento. Vale salientar que existe uma forte ligação da endometriose com as doenças psicossomáticas, envolvendo, dessa forma, uma intensa relação entre corpo e mente na sua origem ${ }^{(3)}$.

Paralelamente, se questiona a relação entre o perfil psicológico e a intensidade da dor relatada pela paciente com endometriose e a dor pélvica crônica, devido à existência de desordem na personalidade pela falta de correlação entre a presença dos sintomas e a extensão da doença. Essa é, portanto, a principal razão para se verificar se existem diferenças no perfil psicológico de determinadas pacientes que estão associadas ao aparecimento dos sintomas, principalmente da dor pélvica crônica ${ }^{(4)}$.

Em suma, à natureza crônica da endometriose, há um potencial impacto sobre a fertilidade e relações íntimas, os atrasos no diagnóstico e a problemática das experiências de cuidados geram um impacto social psicológico da doença sobre as mulheres, sendo um fator digno de atenção. Além disso, há custos muito significativos associados à doença $\mathrm{a}^{(5)}$.

Diante do exposto, este estudo busca compreender a relação existente entre a ocorrência de endometriose e o sofrimento psíquico presente nas mulheres portadoras da doença.

\section{MÉTODOS}

O presente artigo faz parte de um estudo transversal e qualitativo intitulado "Narrativas de dor e sofrimento: histórias de vida de mulheres com endometriose". A coleta de dados foi realizada no período de setembro de 2016 a janeiro de 2017, em um hospital terciário, referência no Estado do Ceará na assistência obstétrica e ginecológica localizado no município de Fortaleza, o qual atua de forma integrada e como suporte aos demais níveis de atenção do modelo de saúde vigente.

A investigação qualitativa aborda uma dimensão simbólica existente nos diversos espaços sociais em que o indivíduo humano se inscreve, permitindo a obtenção de descrições detalhadas de situações com o objetivo de 
compreender os indivíduos em seus próprios termos, obrigando o pesquisador a ter flexibilidade e criatividade no momento de coletá-los e analisá-los ${ }^{(6)}$.

A coleta de dados se deu através de entrevistas narrativas. A relevância das entrevistas narrativas na pesquisa qualitativa importa na contribuição que este instrumento fornece para a compreensão das estruturas processuais dos cursos de vida ou trajetórias dos sujeitos pesquisados. $O$ ato de rememorar e a narração da experiência vivenciada de forma sequencial permitem acessar as perspectivas particulares de sujeitos de forma natural ${ }^{(7)}$.

As onze participantes do estudo foram recrutadas dentro do universo das mulheres com endometriose por ocasião do seu primeiro atendimento no ambulatório de endometriose da Maternidade Escola Assis Chateubrian (MEAC) após a confirmação diagnóstica. Participaram desta pesquisa mulheres maiores de 18 anos diagnosticadas com endometriose. Foram excluídas mulheres com diagnóstico de outras patologias crônicas (além da endometriose) que não possuíam relevância para o estudo.

Após leitura e transcrição minuciosa de todas as entrevistas coletadas das participantes com seu consentimento informado e aplicação dos critérios de inclusão e exclusão, extraiu-se uma categoria relevante para análise, abordando a forte relação da endometriose com o sofrimento psíquico apresentando pelas pacientes.

Na pesquisa científica, a categorização é um modo de encontrar unidade na diversidade e produzir explicações e generalizações através de agrupamentos de elementos, ideias ou expressões em torno de um conceito que expressa a realidade estudada, o que norteia a interpretação dos dados. Portanto, para análise das entrevistas, foi utilizada a técnica de análise temática(6), que consiste em encontrar os núcleos de sentido que compõem uma comunicação. Neste método, a frequência com que os sentidos surgem nas falas das entrevistadas possui um significado para $o$ objeto analítico visado. Para operacionalização do estudo, seguiu-se as etapas pré-análise, exploração do material e tratamento dos resultados obtidos e interpretação(6).

O estudo foi submetido ao Comitê de Ética em Pesquisa da Universidade de Fortaleza e aprovado sob o Parecer $n^{\circ}$ 1.843.153. Respeitaram-se os preceitos éticos de acordo com a Resolução 466/12 do Conselho Nacional de Saúde que regulamenta os aspectos éticos e legais da pesquisa em seres humanos, mantendo-se o anonimato. Em vista disso, as participantes receberam por pseudônimo a letra "A", seguida de numeração arábica.

\section{RESULTADOS E DISCUSSÃO}

A mulher com endometriose pode enfrentar o sofrimento psíquico e a dor psicológica em diferentes graus de intensidade, considerando os outros sintomas clínicos. Outra consequência desse quadro patológico é o comprometimento da qualidade de vida das portadoras. Normalmente, a mulher, ao vivenciar essas mudanças e experimentar esses sentimentos, pode desenvolver quadros de depressão que, quando não são corretamente diagnosticados e tratados, interferem no tratamento da endometriose, agravando-a com o decorrer do tempo ${ }^{(8)}$.

"Eu menstruei e as cólicas voltaram e elas não foram mais embora e as cólicas são umas coisas tremendas, que só a portadora de endometriose entende do que eu estou falando." (A2)

"A médica dizia que era normal, a professora achava que eu estava enrolando e minha mãe começou a achar que era frescura. Você não tem noção, não, do inferno que era. Inferno." (A10)

A endometriose, além do sofrimento físico causado pelos sintomas, provoca um impacto negativo na vida da mulher, interferindo na sua relação com a família, nas relações afetivas, no seu rendimento e desempenho das atividades profissionais, a doença também restringe e modifica o convívio diário da mulher com suas rotinas até então estabelecidas, limitando a sua qualidade de vida, o que reflete, principalmente, na sua autoestima ${ }^{(9)}$ :

"Eu sou uma pessoa muito ansiosa, eu tenho ansiedade." (A2)

"Nem um movimento, eu sem conseguir fazer movimentos de nada, eu só, a tendência era só ficar deitada entendeu? Até passar as dores." (A4)

A busca incessante pelos serviços de saúde no tratamento das dores incapacitantes são condições que contribuem para a alteração do humor da mulher. A demora no diagnóstico e na intervenção levam à mulher a frustração e, frequentemente, à reação estressante. A experiência traumática dessas pacientes quando relatam seus sintomas aos profissionais de saúde gera efeitos negativos nos comportamentos diários. Tais efeitos se prolongam com o passar do tempo, tornando-as frágeis, inoperantes e sem condições para controlar a intensidade de seu problema:

"Eu estava sempre no hospital do lado da minha casa. Mas minha dor no começo passava com Buscopam ${ }^{\circledR}$ e depois só passava com Trama/®. Aí o médico não quis mais passar porque disse que eu estava era viciada, 
que cólica é normal e que eu estava era viciada em remédio." (A11)

"Porque é chato, a pessoa parece que não está levando a sério. Uma enfermeira que era amiga da minha mãe perguntou se eu não estava querendo chamar a atenção, porque meus pais estavam se separando." (A9)

A negligência no manejo da dor e a depreciação do corpo por ocasião da doença é outro aspecto do sofrimento psíquico vivido por essas mulheres, ocorrendo, assim, uma banalização das características que são relatadas com classificações pejorativas pelos profissionais de saúde. Quando o indivíduo sofre é porque há alguma coisa, sua fala não deve ser negada, desqualificada ou colocada em dúvida. As mulheres se revoltam pela não compreensão ou se insurgem contra a incompetência dos profissionais em traçar um diagnóstico e tratar. Tal fato as leva ao sofrimento ${ }^{(10)}$ :

"Eu passei dez dias em casa, infecionada, sangrando muito e eu pensava que era normal e quando foi um dia eu, não é normal, vim pra emergência, aí quando eu cheguei foi que os médicos mandaram (eu) sabe, o sangue estava podre mesmo, aí foi onde eu sofri, viu, também, eles começaram a limpar quando eu subi lá." (A6)

"Olha, a pessoa com dor e a outra vem dizer que eu estava querendo chamar a atenção, é de morrer não é não? Ai era chato. Os médicos, já me conheciam também e já brincavam perguntando se era o de sempre. $O$ de sempre era Profenid $\circledast$, Buscopam $\circledast$ e Plasil $\circledast$ na veia." (A9)

A depressão é bastante comum em pacientes com endometriose. Muitas vezes, a própria manifestação da dor crônica está relacionada ao grau de depressão e ansiedade presente na mulher ${ }^{(7,11,12)}$. Mesmo após o estabelecimento do diagnóstico e a instituição do tratamento para a doença, os fatores psicológicos que cursam, como parte da síndrome dolorosa, são: a mudança no humor e a ansiedade, os quais contribuem diretamente para melhorar ou piorar o estado de saúde da mulher.

"Há quatro anos eu trato a endometriose e foi uma doença que me machucou muito, que eu tive (que) graves problemas na minha vida, principalmente psicológicos, que até hoje, eu sofro com eles." (A1)

As mulheres necessitam de uma abordagem holística para visibilidade dos sintomas, precisando de um suporte emocional para lidar com a doença. A doença pode ser intensificada ou enfraquecida de acordo com o estado emocional presente na mulher, afetando-a não só fisicamente, mas também todo o seu convívio familiar e social, a sua vida sexual e, principalmente, a relação conjugal com o parceiro, em função do comprometimento da patologia( ${ }^{(13)}$ :

"Eu gostava da escola antes. Depois deixei de gostar. Eu gostava da minha mãe antes. Agora só tenho mágoa." (A10)

"Meu casamento é ótimo. Meu marido é carinhoso. Mas ele não entendia muito que eu não queria, porque estava com dor. Ele começou a ter ciúme, achando que eu estava traindo ele. E aí era ruim por que além da dor eu estava com raiva e aí era meio forçado. Eu só fazia porque tem que fazer." (A9)

"Meu primeiro namorado que eu fiz sexo me chamou de doida porque doeu muito e eu gritei na hora. Ele me chamou de histérica." (A10)

Os sintomas psíquicos geram sofrimento na paciente de modo permanente e progressivo. A avaliação e o tratamento dos sintomas psíquicos são simples, de baixo custo e podem ser independentes de especialistas. Salienta-se ainda que as terapias se mostram eficazes no tratamento, propiciando melhorias significativas ${ }^{(14)}$. Essa condição ginecológica crônica influencia os planos futuros da mulher, sendo estes condicionados pelo medo, o qual inclui o desejo da gravidez esbarrado pela infertilidade ocasionada pela patologia:

"O meu maior medo que eu tive foi de não poder engravidar, mas hoje, eu descobri que eu posso e que está tudo bem comigo e isso é o que me fortalece todo dia, eu peço pra todo mundo ter mais cuidado consigo." (A1)

"(...) claro que de vez em quando eu choro, porque não posso ter um filho, porque não posso engravidar, por isso, por aquilo e muito mais porque tem um tratamento muito grave e eu já vejo, estou chegando quase aos trinta e eu não vou mais querer, é opção minha não querer mais não é? Porque nunca deu certo, então pra mim eu não quero mais." (A8)

O cuidado à mulher com endometriose não se resume apenas no manejo dos sintomas da patologia orgânica, mas significa também avaliá-la integralmente, o que inclui o seu estado emocional. Sendo assim, faz-se necessário identificar a relação dos sintomas psíquicos com o desenvolvimento da endometriose, uma vez que podem alterar o curso natural da doença. O sentimento positivo relatado foi o alívio em tomar conhecimento de um diagnóstico 
que explicava sua condição. O tratamento é uma nova fase que evoca outros sentimentos nas pacientes e também traz efeitos negativos para algumas mulheres, sendo que algumas o abandonam ${ }^{(15)}$.

"Vou dizer que parece loucura isso. Mas foi a melhor notícia do mundo eu saber que tinha endometriose. Eu achava que era louca, que era fresca, que era fraca, que eu tinha vindo com defeito, não é." (A10)

"Fiz uso de todos os anticoncepcionais que eles já conheciam, porque eu comecei a ter reações aos anticoncepcionais, dores no estômago, ânsia e vômito, eu comecei a perder peso, então eu comecei a ter reações e aí com tempo eu fiquei adolescente, fiquei revoltada, disse que não ia mais cuidar da endometriose." (A8)

A endometriose se trata de uma doença ginecológica crônica caracterizada por sintomas consideravelmente comprometedores observados através dessas narrativas. Nesse sentido, é importante a busca por estratégias para promoção da saúde, dando o suporte emocional para lidar com uma doença que pode ser não somente debilitante, mas socialmente, sexualmente e psiquicamente devastadora e que provoca incertezas de um diagnóstico que necessita de apoio humano dos profissionais para o restabelecimento da qualidade de vida de todas essas mulheres.

\section{CONSIDERAÇÕES FINAIS}

$\mathrm{Na}$ endometriose, os quadros frequentes de dor, da infertilidade e do diagnóstico tardio são razões que contribuem para que as pacientes elevem os níveis de ansiedade e depressão. Apesar de a ansiedade e a depressão serem transtornos clinicamente diferentes, é comum as pessoas apresentarem ambos concomitantemente.

A expressão da qualidade de vida ligada à saúde é deliberada como o valor atribuído à duração da vida quando esta é transformada pela percepção de limitações físicas, psicológicas, funções sociais e oportunidades motivadas pela doença, tratamento e outros agravos. Em concordância com esse sentido sobre a percepção da saúde, as funções sociais, psicológicas e físicas das mulheres com endometriose necessitam ser repensadas e reconfiguradas.

Tendo em vista a diversidade de queixas, atualmente, busca-se um tratamento multiprofissional que atenda às demandas físicas e psicológicas das mulheres com endometriose. Já existe, por parte dos profissionais de saúde, a percepção da necessidade de suporte psicológico para essas mulheres, sendo esta abordagem imperativa para o tratamento.

Portanto, conclui-se que a qualidade de vida deve ser um aspecto observado no tratamento de pacientes com endometriose, sobretudo quando se considera a influência dos aspectos psíquicos no tratamento da doença biológica.

\section{CONFLITOS DE INTERESSE}

Não há conflitos de interesse na realização deste trabalho.

\section{REFERENNCIAS}

1. Facchin F, Saita E, Barbara G, Dridi D, Vercellini P. Free butterflies will come out of these deep wounds: a grounded theory of how endometriosis affects women's psychological health. J Health Psychol. 2018;23(4):538-549.

2. Donatti L, Ramos DG, Andres MP, Passman LJ, Podgaec S. Patients with endometriosis using positive coping strategies have less depression, stress and pelvic pain. Einstein. 2017;15(1):65-70.

3. Moradi M, Parker M, Sneddon A, Lopez V, Ellwood D. Impact of endometriosis on women's lives: a qualitative study. BMC Womens Health. 2014;14:123.

4. Lorençatto $C$, Vieira MJN, Pinto CLB, Petta CA. Avaliação da frequência de depressão em pacientes com endometriose e dor pélvica. Rev Assoc Med Bras. 2002;48(3):217-221.

5. Weller W, Zardo SP. Entrevistas narrativas com especialistas: aportes metodológicos e exemplificação. Rev Educ Contemp. 2013;22(40):131-143.

6. Minayo, MCS. O desafio do conhecimento: pesquisa qualitativa em saúde. 13a ed. São Paulo: Hucitec; 2013.

7. Culley L, Law C, Hudson N, Denny E, Mitchell H, Baumgarten M; et al. The social and psychological impact of endometriosis on women's lives: a critical narrative review. Hum Reprod Update. 2013;19(6):625-639.

8. Flores RC, Lara EB, Corral LCQ, Chaib RAI, Pérez LO, González Díaz OA; et al. Quality of life in women with 
endometriosis pelvic pain treated with the levonorgestrel-releasing intrauterine system. Open J Obstet Gynecol. 2015;5(3):167-72.

9. Lorençatto C, Vieira MJN, Marques A, Benetti-Pinto CL, Petta CA. Avaliação de dor e depressão em mulheres com endometriose após intervenção multiprofissional em grupo. Rev Assoc Med Bras. 2007;53(5):433-438.

10. Bento PASS, Moreira MCN. A experiência de adoecimento de mulheres com endometriose: narrativas sobre violência institucional. Ciênc. Saúde Colet. 2017; 22(9):3023-3032.

11. Silva MPC, Medeiros BQ, Trovó de Marqui AB. Depressão e ansiedade em mulheres com endometriose: uma revisão crítica da literatura. Interação psicol. 2016;20(2):226-233.

12. Figueiredo J, Nascimento R. Avaliação da qualidade de vida de pacientes portadoras de endometriose após inserção do Sistema Intra-Uterino Liberador de Levonorgestrel (SIU-LNg). ACM Arq Catarin Med. 2008;37(4):2026.

13. Minson FP, Abrão MS, Sardá J Jr., Kraychete DC, Podgaec S, Assis FD. Importância da avaliação da qualidade de vida em pacientes com endometriose. Rev Bras Ginecol Obstet. 2012;34(1):11-15.

14. Vila ACD, Vandenberghe L, Silveira NA. A vivência de infertilidade e endometriose: pontos de atenção para profissionais de saúde. Psicol Saúde Doenças. 2010;11(2):219-228.

15. Rodrigues PSC, Silva TASM, Souza MMT. Endometriose - importância do diagnóstico precoce e atuação da enfermagem para o desfecho do tratamento. Rev. Pró-UniverSUS. 2015;6(1):13-16.

\section{Endereço para correspondência:}

Luis Adriano Freitas Oliveira

Universidade de Fortaleza - UNIFOR

Programa de Pós-Graduação em Saúde Coletiva

Av. Washington Soares, 1321, Bloco S, Sala 1

Bairro: Edson Queiroz

CEP: 60.811-905 - Fortaleza - CE - Brasil

E-mail: adrianojs03@gmail.com 